\title{
The Interaction of Single Walled Carbon Nanotube (SWCNT) with Phospholipids Membrane: in Point View of Solvent Effect
}

\author{
AKBAR ELSAGH ${ }^{1 *}$, HAMIDREZA JALILIAN ${ }^{2}$ and ALI R. ILKHANI ${ }^{3}$ \\ ${ }^{1}$ Department of Chemistry, North Tehran Branch, Islamic Azad University, Tehran, Iran. \\ 2Department of Chemistry, Gorgan Branch, Islamic Azad University, Gorgan, Iran. \\ ${ }^{3}$ Department of Chemistry, Yazad Branch, Islamic Azad University, Yazad, Iran. \\ ${ }^{*}$ Corresponding author:Akbarelsagh @yahoo.com
}

http://dx.doi.org/10.13005/ojc/310124

(Received: November 30, 2014; Accepted: January 10, 2015)

\begin{abstract}
In this research, we have studied the structural properties of phospholipids, surrounding single-walled carbon nanotube (SWCNT, by using ab-inition and molecular dynamics simulation. Carbon nanotubes (SWCNTs) are very common in medical research and are being highly studied in the fields of biosensing methods for disease treatment and efficient drug delivery and health monitoring. The transportation of SWCNT through the cell membrane widely investigated because of many advantages. Because of the differences among force fields, the energy of a molecule calculated using two different force fields will not be the same. In this study difference in force field illustrated by comparing the energy of calculated by using force fields, MM+, Amber and OPLS. The quantum Mechanics (QM) calculations were carried out with the GAUSSIAN 09 program based on density functional theory (DFT) at B1LYP/6-31G* level. In our recent study the electronic structure of open-end of SWCNT and transportation of SWCNT through the phospholipids in skin cell membrane have been discussedfor both vacuum and solvent media.
\end{abstract}

Key words: Solvent effect, Phospholipids membrane, Single walled carbon nanotube.

\section{INTRODUCTION}

Nature provides us with a very large number of channels or nano-pores embedded in cell membranes. The function of channels is to allow selectivity and specificity for a Variety of molecular species transport across the cell membrane. These channels,included : a)ligand-gated channels) voltage-gated channels, c)Second messenger gated channels, d)mechanic sensitive channels) Gap junctions: porins not gated ${ }^{1-7}$.
The discovery of carbon nanotubes (CNT) in 1991 heralded the era of nanoscience and nanotechnology ${ }^{8-11}$.

Nanotubes of carbon and other materials, due to their electronic, optical and mechanical properties find applications in several fields ${ }^{12-18}$.

The space available inside the nanotube enables it to match phospholipids outside. This makes the carbon nanotube an ideal medium for storing high energy materials ${ }^{19-21}$. 
Carbon nanotubes (CNTs) since their discovery ${ }^{22}$ have been the focus of scientific research due to their outstanding chemical, mechanical and electrical properties. Single walled carbon nanotubes (SWCNTs) are of particular interest because of their size and superior electrical properties ${ }^{23}$. SWCNTs have been used to realize many molecular scale electronic devices ${ }^{24-29}$.

Membranes are asymmetric structures. The choline-containing phospholipids are located mainly in the outer molecular layer. This asymmetric distribution is maintained by an ATP-dependent protein which specifically Trans-locates phosphatidyl-ethanolamine (and phosphatidylserine) to the inside of the plasma membrane ${ }^{30-33}$.

All major lipids in membranes contain both hydrophobic and hydrophilic regions and are therefore termed amphipathic. Dipalmitoylphosp hatidylcholine (DPPC) anddimyristoylphos phatidylcholine (DMPC) are taken as phospholipids with an equal polar heads and with the difference in the length of hydrocarbon chain $^{34,35}$. These two molecules have saturated fatty acid tail groups ${ }^{36-39}$.

On a molecular level it has been of interest to explore to what extent PC head groups differ with respect to molecular conformation, lateral interactions, and dipole arrangements and how these features affect the properties and topology of the membrane surface ${ }^{40-44}$.

There are two types of CNTs: single-walled nanotubes (SWCNTs) and multi-walled nanotubes $(\mathrm{MWCNTS})^{45}$; that they have three conformation:

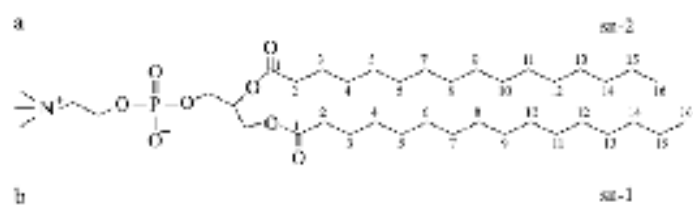

Fig.1: DPPC and DMPC molecule

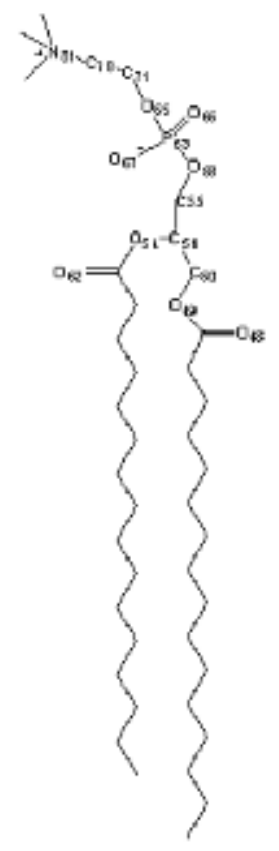

Fig. 2: DPPC armchair $(n, n)$, zigzag $\left(n,,_{0}\right)$ and chiral $(n, m)$ these conformations have individual properties ${ }^{46-50}$.

SWCNTs have been considered as the leading candidate for nanodevice applications because of their one-dimensional electronic bond structure, molecular size, and biocompatibility, controllable property of conducting electrical current and reversible response to biological reagents hence SWCNTs make possible bonding to polymers and biological systems such as DNA and carbohydrates ${ }^{51-57}$.

\section{Computational Methods}

All calculations have done by ab initio at the Hartree-Fock (HF) level of theory Gaussian 98 package[58]. Four basis sets have used, namely the sto-3G ,3-21G,6-31G and 6-31G*. The geometry of DPPC, DMPC have full optimized at the RHF/6-31G*, 6-31G, 3-21G and STO-3G levels of the theory in the gas phase ${ }^{59-61}$. 


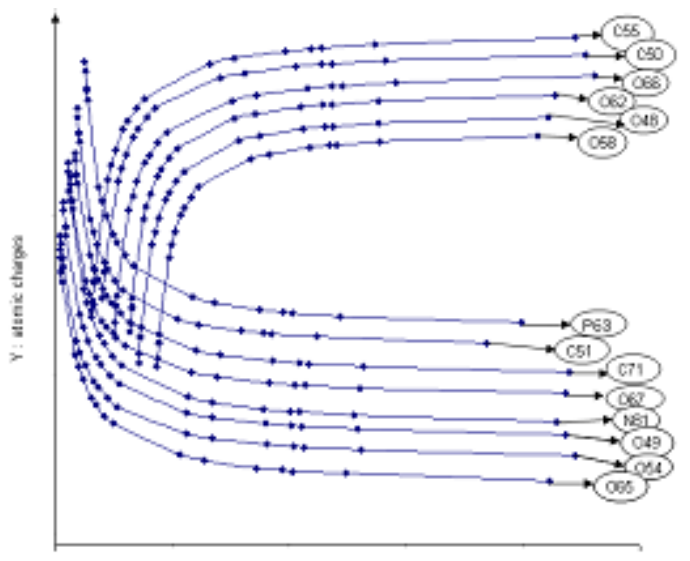

Fig. 3: The charge changing due to each hydrophobic chain of the DPPC

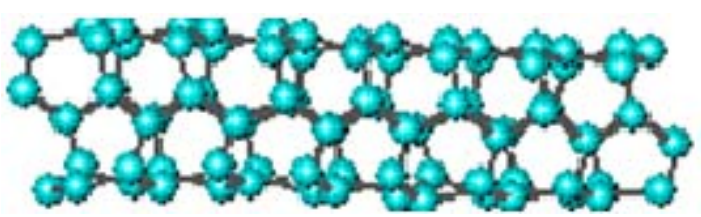

a) $\mathrm{C}_{84}(3,7)$-Water

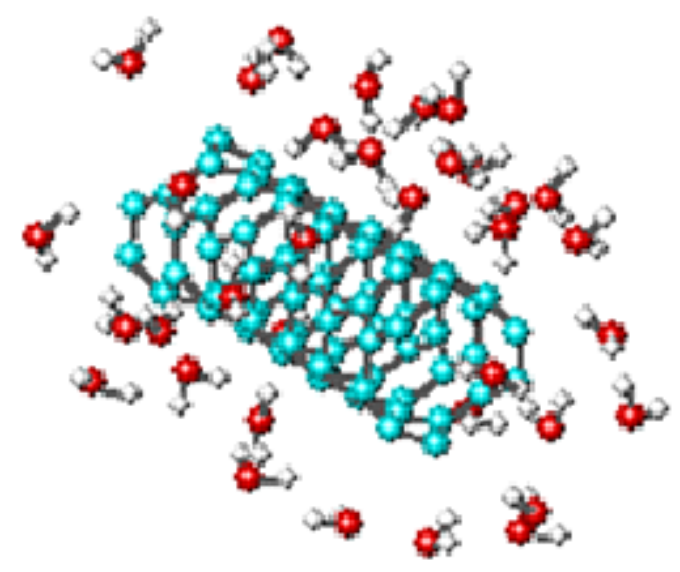

a) $\mathrm{C}_{84}$ (3,7)-Water-Methanol

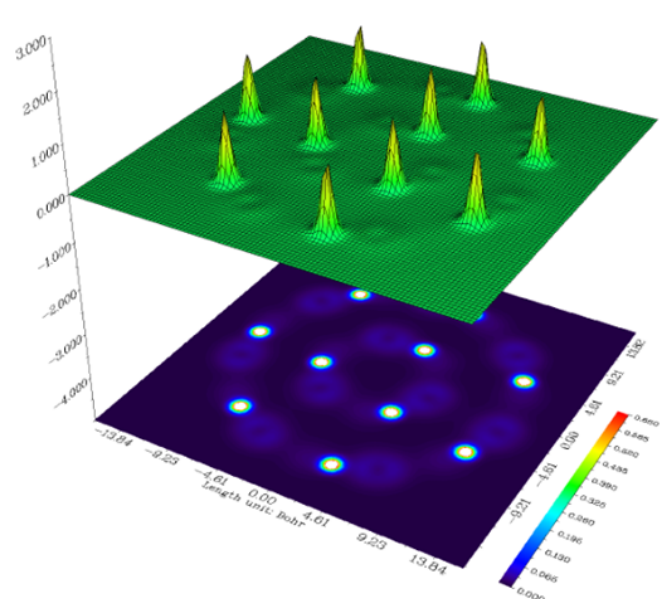

Fig. 4: The electron density of the nanotube

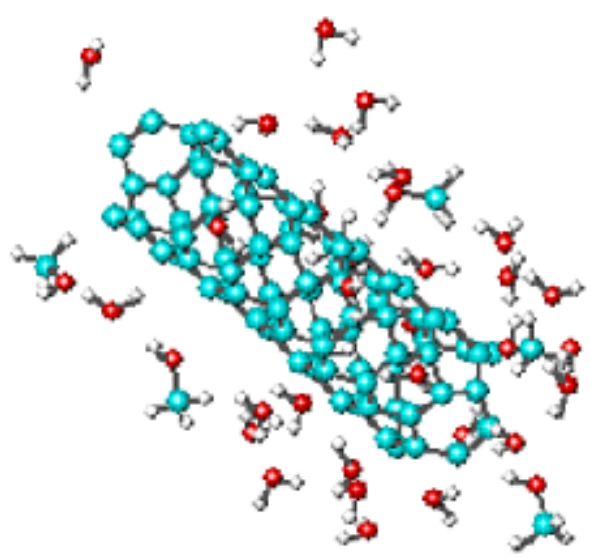

b) $\mathrm{C}_{84}(3,7)$-Water

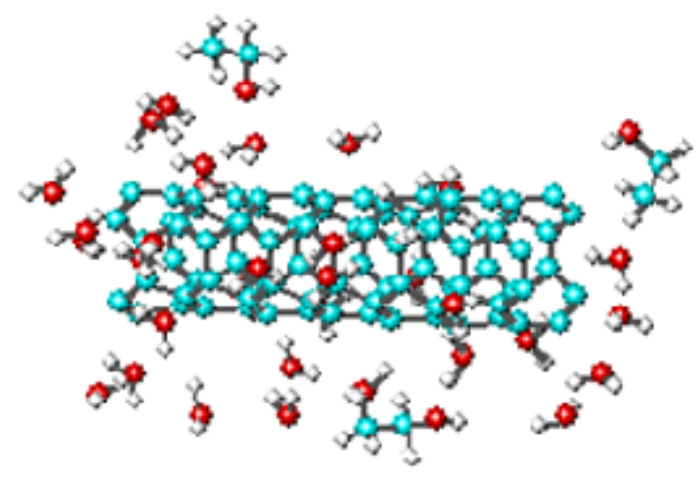

b) $\mathrm{C}_{84}(3,7)$-Water-Methanol

Fig. 5: The Nano tubes in various solvent of water and methanol 
The most important dihedral angle of these molecules (DPPC and DMPC) is chosen and

The energy, dipole moment, and atomic charges of 15 important atoms have scanned within 180 degrees rotation. In this manner after the optimization of total molecules, important dihedral angle of these molecules has rotated 15 degrees at every time $62-64$.

The effects of the solvent polarization are described in terms of proper QM operators to be added to the Hamiltonian of the isolated system then, the salvation calculations have performed using Onsager method at $\mathrm{HF} / 6-31 \mathrm{G}^{*}$. For Onsager model, it does require values of volume $\left(a_{0}\right)$ of the molecule and the dielectric $(\varepsilon)$ of solvent. The volume of DPPC and DMPC molecules was obtained using the "volume" keyword. The Onsager-SCRF was that it permitted one to directly exploit almost all of the computational facilities of the Gaussian packages .For this reason, and for its very limited computational cost, it is still in use by people not requiring an accurate description of salvation effects but just a guess or a qualitative correction to the values obtained for the isolated molecule. Users must be aware of the limitations of the approach, of the unphysical deformation of the solute charge distribution it may induce, and of other shortcomings specific of the approach, such as the lack of salvation for solutes with zero permanent dipole ${ }^{65-66}$.

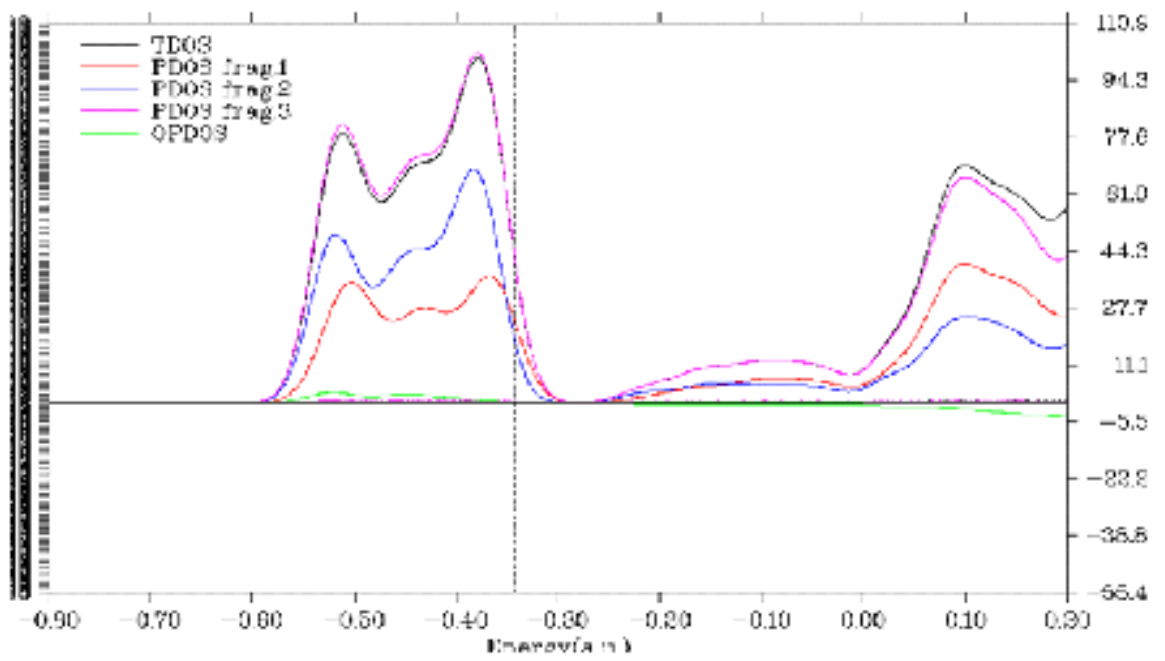

Fig. 6: Density of state

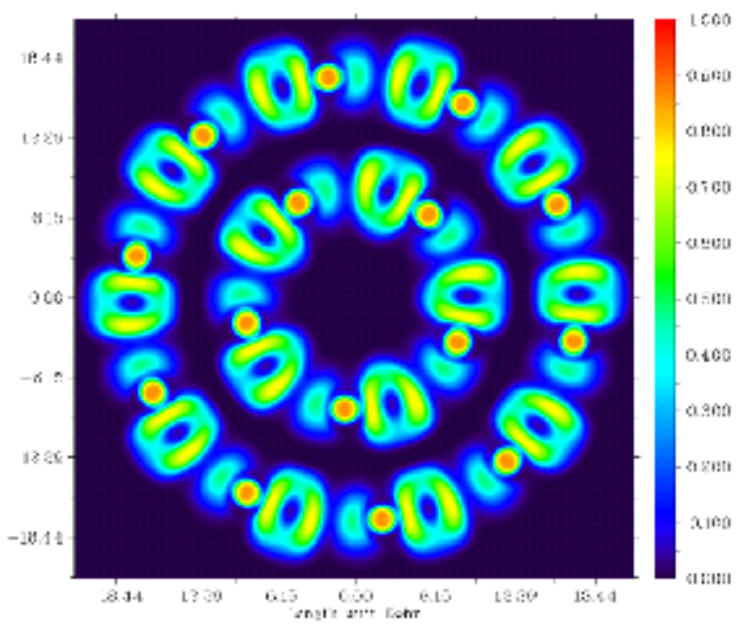

Fig. 7: Contour map of density state for solvents 
In the Onsager method, the solute molecule is placed in a spherical cavity of radius $a_{0}$ surrounded by a continuum with constant dielectric properties ${ }^{67}$. A dipole in the molecule will induce a dipole in the medium, and the electric field applied by the solvent dipole will in turn in interact with the molecular dipole leading to net stabilization. The molecular geometrics have obtained via HF/6-31G* level optimization in the gas phase and have rotated and then any one separately havebeen placed in the solvents ${ }^{67,68}$.

\section{RESULT AND DISCUSSION}

Dipalmitoylphosphatidylcholine (DPPC) and dimyristoylphosphatidylcholine (DMPC) molecules have chosen as startingstructures for gas phase (Fig1).
The DPPC and DMPC zwitter-ionic are found to be unstable in the gas phase when have optimized at HF/3-21G, 6-31G and 6-31G* level.The obtained result from optimization and stabilization parameters are shown in table1.

Table 1: Conformational energy of DPPC and DMPC obtained by geometry optimization for different basis set

\begin{tabular}{lll}
\hline Basis set & $\begin{array}{l}\text { E/Kcal.mol-1 } \\
\text { DPPC }\end{array}$ & $\begin{array}{l}\text { E/Kcal.mol-1 } \\
\text { DMPC }\end{array}$ \\
\hline Sto-3G & -1583811.25 & -148406.13 \\
3-21G & -1594584.734 & -149458.5 \\
6-31G & -1602683.01 & -1505418.6 \\
4-31g & -1604567.05 & -1505341.9 \\
6-31G* & -1603386.341 & -1505422.7 \\
6-311++G** & -1605567036 & -160552.9 \\
\hline
\end{tabular}

\section{REFERENCES}

1. L.K.Kaczmarek, T.M. Perney, curr. opin. cell. Biol. 1991, 3, 663.

2. D.A. Doyle, J. Moraiscabral, Science 1998, $280,69$.

3. Kratschmer W, Lamb LD, Fostiropoulos K, Huffman DR.Solid C60-a new form of carbon. Nature 1990, 347, 354.

4. MajidMonajjemi, Robert Wayne, JrandJames E. Boggs, Chemical Physics , 2014, 433,1-11.

5. T. Ardalan , P. Ardalan\& M. Monajjemi,Fullerenes, Nanotubes, and Carbon Nanostructures, 2014, 22, 687-708.

6. M. Monajjemi, S. Ketabi, M. HashemianZadeh, and A. Amiri, Biochemistry (Moscow), 2006, 1, Suppl 1, S1S8.

7. M. Monajjemi, S. Ketabi, A. Amiri, Russian Journal of physical chemistry , 2006, 1, 5562.

8. S. lijima, Nature 1991, 354, 56.

9. M. Monajjemi, Struct Chem., 2012, 23, 551580.

10. M. Monajjemi , N. Karachi \& F. Mollaamin, Fullerenes, Nanotubes, and Carbon Nanostructures, 2014, 22, 643-662.
11. M. Monajjemi, J.E Boggs, J. Phys. Chem A. 2013, 117, 1670.

12. M. Monajjemi, L. Mahdavian, F. Mollaamin and $\mathrm{M}$. Khaleghian ,Interaction of $\mathrm{Na}, \mathrm{Mg}$, $\mathrm{Al}$, Si with Carbon Nanotube (CNT): NMR and IR Study Russian Journal of Inorganic Chemistry, 2009, 54(9), 1465-1473.

13. F. Mollaamina, Z. Varmaghani, and M. Monajjemi.Dielectric effect on thermodynamic properties in vinblastine by DFT/Onsager modeling, Physics and Chemistry of Liquids, , 2011, 49(3), 318-336.

14. Monajjemi $M$, Lee $\mathrm{V} S$, Khaleghian $M$, Honarparvar B, Mollaamin F. Theoretical Description of Electromagnetic Nonbonded Interactions of Radical, Cationic,and Anionic $\mathrm{NH} 2 \mathrm{BHNBHNH} 2$ Inside of the B 18 N18Nanoring. J. Phys. Chem. C. 2010, 114, 15315-15330.

15. M. Monajjemi, L. Mahdavian, F.Mollaamin. Characterization of nanocrystallinecylicon germanium film and nanotube in adsoption gas by montecarlo and langevin dynamic simulation Bull. Chem. Soc. Ethiop, 2008, 22(2), 1-10.

16. M. Monajjemi, M. Seyed Hosseini\& F. 
Mollaamin, Fullerenes, Nanotubes, and Carbon Nanostructures, 2013, 21, 381-393.

17. F. Mollaamin , M. Monajjemi\& J. Mehrzad, Fullerenes, Nanotubes, and Carbon Nanostructures, 2014, 22, 738-751.

18. M. Monajjemi; H. Chegini; F. Mollaamin; P. Farahani, Fullerenes, Nanotubes, and Carbon Nanostructures, 2011, 19, 469-482.

19. H.A. Rachid, A. Hu, V. Timoshevskii, Y. Song, L.S. Lussier, Phys. Rev. Lett. 2008, 100, 204.

20. Majid Monajjemi, Fatemeh Mollaamin , Tahereh Karimkeshteh, J. Mex. Chem. Soc. 2005, 49(4), 336-340

21. V.S.Lee,P.Nimmanpipug, F. Mollaamin, N. Kungwan, S.Thanasanvorakun, and $M$. Monajjemi, Russian Journal of Physical Chemistry A, 2009, 83(13), 2288-2296.

22. lijima $S$. Helical microtubules of graphitic carbon. Nature 1991, 354, 56.

23. Odom TW, Huang JL, Kim P, Lieber CM. Atomic structure and electronic properties of single-walled carbon nanotubes. Nature 1998, 39, 62.

24. Postma HWC, Teepen T, Yao Z, Grifoni M, Dekker C. Carbon nanotube single-electron transistors at room temperature. Science 2001, 293, 76.

25. M. Monajjemi, A. Sobhanmanesh, \& F. Mollaamin, Fullerenes, Nanotubes, and Carbon Nanostructures, 2013, 21: 47-63.

26. F. Mollaamin, K. Shahani pour, K. Shahani pour, A. R. Ilkhani, Z. Sheckari, and M.Monajjemi, Russian Chemical Bulletin, International Edition, 2012, 61(12).

27. M. Monajjemi, F. Mollaamin, J Clust Sci, 2011, 22, 673 .

28. H.Yahyaei , M. Monajjemi , H. Aghaie, and K. Zare, Journal of Computational and Theoretical Nanoscience, 2013, 10(10), 2332-2341.

29. M. Monajjemi\& M. Falahati\& F. Mollaamin, Ionics 2013, 19:155-164

30. A.Zachowski, E.Favre, S.Cribier, P.Hevre', P. F.Devaux, J.Biochemistry 1986, 25, 25852590.

31. Monajjemi,M. Honarparvar, B. H. Haeri, H. Heshmat, M. Russian Journal of Physical Chemistry C., 2006, 80(1): S40-S44.

32. A. Tahan and M. Monajjemi, Acta Biotheor 2011, $59: 291-312$
33. M. Monajjemi, R. Sayyadi, G. Ghasemi, Kh.Lalateh, A. Nouria, F. Naderi,Main Group Metal Chemistry, 2005, 28(5), 247-263

34. M. A. Kiselev,P. Lesieur, A. M. Kisselev and M. Ollivon, Ice Formation in Model Biological Membranes in the Presence of Cryoprotectors, Nuclear Instruments \& Methods in Physics Research ., 2000, A 448, 225-260.

35. H. yun, Y.w. Choi,N. J. Kim.and D. Sohn., Physicochemical properties of Phosphatidylcholine (PC) Monolayers with Different Alkyl Chains,at the Air/Water Interface., Bull. Korean hem. Soc. 2003, 24(3), 337.

36. J. Landin and I. Pascher, Effect of a Polar Environment on the Conformation of Phospholipid Head Groups Analyzed with the Onsager Continuum Solvation Model. J. Phys. Chem. A 1997, 101, 2996-3004

37. M. Monajjemi, E. Rajaeian, F. Mollaamin, F. Naderi, and S. Saki., Phys. Chem. Liq. 2008, 46, 3299.

38. M.Monajjemi, M.Heshmat, H. Aghaei, R. Ahmadi, K.Zare,Bulletin of the Chemical Society of Ethiopia 21 (1), pp. 111-116, 2007

39. Monajjemi, M., Aghaie, H.,Naderi, F , Biochemistry (Moscow) 2007, 72(6),799-804.

40. A.J. Robinson, W.G. Richards, P.J. Thomas, M.M.Hann, Head group and chain behavior in biological membranes: a molecular dynamics computersimulation. Biophys $\mathrm{J}$. 1994, 67(6), 2345-2354

41. MONAJJEMI M, Zare K, Gharib F, Journal of Chemical and Engineering Data 1995, 40(2): 419-422.

42. M. Monajjemi, F. Naderi, F. Mollaamin, and M. Khaleghian, J. Mex. Chem. Soc. 2012 , 56(2), 207-211

43. Monajjemi M, Azizi Z, Ghavami M, Russian Journal of Inorganic Chemistry 2003, 48(10): 1551-1559.

44. M. Monajjemi, F. Naderi, H. Aghaie, M. Yari, D.N. Mansoor, S. Afsharnezhad, F. Mollaamin, Research Journal of Chemistry and Environment 2007, 11(2), 56-62.

45. M. Foley, Cheap Tubes, 2005.

46. B. Gojman, H. Hsin, J. Liang, N. Nezhdanova and J. Saini, 2004.

47. F. Mollaamin , F. Najafi , M. Khaleghian , B. 
KhaliliHadad\& M Monajjemi , Fullerenes, Nanotubes, and Carbon Nanostructures, 2011, 19(7), 653-667.

48. H. Yahyaei\& M. Monajjemi,Fullerenes, Nanotubes, and Carbon Nanostructures, 2014, 22(4): 346-361.

49. M. Monajjemi, and M. Ahmadianarog, Journal of Computational and Theoretical Nanoscience 2014, 11(6), 1465-1471

50. B. Ghalandari, M. Monajjemi, and F. Mollaamin, Journal of Computational and Theoretical Nanoscience, 2011, 8, 12121219.

51. T. Ramanathan, F. T. Fisher, R. S. Ruoff and L. C. Brinson, Chem mater, 2005, 17, 1290.

52. M. Monajjemi, M.H.Razavian, F.Mollaamin, F.Naderi, B.Honarparvar, Russian Journal of Physical Chemistry A 2008, 82(13), 22772285.

53. M. Monajjemi, J. Najafpour\& F. Mollaamin., Fullerenes, Nanotubes, and Carbon Nanostructures 2013, 21(3): 213-232.

54. F. Mollaamin and M. Monajjemi, Journal of Computational and Theoretical Nanoscience , 2012, 9(4), 597-601.

55. M. Monajjemi, R. Faham\& F. Mollaamin, Fullerenes, Nanotubes, and Carbon Nanostructures, 2012, 20, 163-169.

56. M. Monajjemi, and F. Mollaamin, Journal of Computational and Theoretical Nanoscience, 2012, 9(12), 2208-2214.

57. M. Monajjemi, H. Baheri, and F. Mollaamin, Journal of Structural Chemistry. 2011, 52(1), 54-59.

58. M.J. Frisch, G.W.Trucks, H.B.Schlegel, G.E. Scuseria, M.A. Robb, J.R Cheeseman, V.G.Zakrzewski,J.A. Montgomery, Jr., R.E. Stratmann, J.C.Burant,'S. Dapprich, J.M. Millam, A.D. Daniels, K.N. Kudin,M.C. Strain, O. Farkas, J. Tomasi, V. Barone, M. Cossi , R.Cammi, B. Mennucci, C. Pomelli, C. Adamo, S. Clifford, J.Ochterski, G. A. Petersson, P.Y. Ayala, Q. Cui, K.Morokuma,
D.K. Malick,A. D. Rabuck, K. Raghavachari, J.B. Raghavachari, J. Cioslowski, J. V. Ortiz, A.G. Baboul,B.B. Stefanov, G. Liu, A. Liashenko, P. Piskorz, I. Komaromi,R. Gomperts, R.L. Martin, D. J. Fox, T. Keith, M. A. Al-Laham, C. Y. Peng, A. Nanayakkara, C. Gonzalez, M.Challacombe, P. M. W. Gill, B. Johnson, W. Chen, M. W.Wong, J. L. Andres, C. Gonzalez, M. Head-Gordon, E. S.Replogle, J. A. Pople. Gaussian, Inc., Pittsburgh PA, 1998.

59. Majid Monajjemi, Chemical Physics, 2013, 425, 29-45.

60. M. Monajjemi , M. Heshmat ,H. H. Haeri, Biochemistry (Moscow), 2006, 71, S113S122.

61. Monajjemi M, Ghiasi R, Abedi A,Russian Journal of Inorganic Chemistry, 2005, 50(3), 382-388.

62. M. Monajjemi, S.Afsharnezhad, M.R. Jaafari, T.Abdolahi,A.Nikosade and H.Monajemi, Russian Journal of physical chemistry $A$, 2007, 2,1956-1963.

63. Monajjemi, M. Ghiasi, R. Ketabi, S., Journal of Chemical Research. 2004, 1: 11-18 .81. Wadt, W. R and Hay, P. J. J. Chem. Phys, 1985, 82, 284.

64. Majid Monajjemi, Chemical Physics 2013, 425, 29-45.

65. Monajjemi M, Chahkandi B, Zare K,Amiri A., Biokhimiya 2005, 70(3), 447-458.

66. Monajjemi, M., Afsharnezhad, S., Jaafari, M. R., Mirdamadi, S., Mollaamin, F. and Monajemi, H., .Chemistry 2008, 17(1): 5569.

67. M.Sundaralingam, Molecular structures and conformations of the phospholipiods and Sphingomyelins. Ann. NY Acad. Sci. 1972, 195, 324-355.

68. Monajjemi M, Chahkandi B, journal of molecular structure-theochem 2005, 714 (1): 43-60. 